\section{Aripiprazol: Therapie der Manie}

\author{
Claus Normann \\ Abtl. Psychiatrie und Psychotherapie der Universitätsklinik \\ für Psychiatrie und Psychosomatik, Freiburg
}

psychoneuro 2003; 30 (3): 160-163

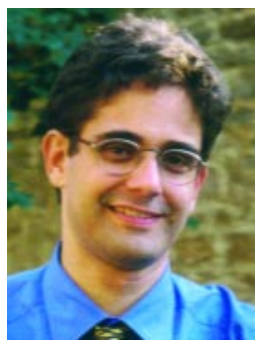

Claus Normann
Nachdem in der Vergangenheit die meisten manischen Patienten mit klassischen Neuroleptika behandelt wurden, werden diese zunehmend von Atypika abgelöst. Insbesondere in Kombination mit Stimmungsstabilisierern bieten atypische Antipsychotika eine effektive und weitgehend sichere Behandlungsoption bei schweren manischen Exazerbationen. Aripiprazol ist ein Atypikum mit einem neuartigen Wirkmechanismus. Die Substanz ist ein partieller DopaminAgonist und verhindert eine dopaminerge Überstimulation, ohne die $D_{2}$-Rezeptoren vollständig auszuschalten. Ein ähnlicher Wirkmechanismus besteht an $5-H T_{1 A}-$ Rezeptoren, $5-H T_{2 A}-R e-$ zeptoren werden hingegen blockiert. Aripiprazol ist in den USA zur Behandlung der Schizophrenie bereits zugelassen, in der EU ist die Zulassung beantragt. In zwei großen DoppelblindStudien wurde Aripiprazol zur Behandlung der akuten Manie untersucht. Dabei zeigte sich eine Überlegenheit gegenüber Plazebo und eine gleichwertige Wirkung verglichen mit Haloperidol. Die Behandlung mit Aripiprazol wurde besser vertragen als mit Haloperidol und es kam zu weniger Therapieabbrüchen. Aripiprazol scheint eine vielversprechende Therapieoption bei bipolarer Störung zu sein.

K lassische Neuroleptika werden immer noch von vielen Klinikern als unverzichtbar zur Therapie der akuten Manie angesehen. In akuten Exazerbationen einer bipolaren Störung werden viele Patienten hochdosiert mit Neuroleptika behandelt, in vielen Fällen wird dieses Behandlungsregime auch noch lange nach Besserung der initialen manischen Symptomatik weitergeführt. Drei Viertel aller Patienten mit einer ersten manischen Episode werden mit Neuroleptika behandelt und bei $25-50 \%$ dieser Patienten wird diese Therapie auch sechs Monate später noch fortgesetzt (16). Darunter kommt es häufig zu extrapyramidal-motorischen Nebenwirkungen mit negativen Effekten auf die Compliance der Patienten; zusätzlich ist zu befürchten, dass durch klassische Neuroleptika der depres- sive Anteil der bipolaren Störung nicht gebessert und eventuell noch verschlimmert wird (3).

Klassische und atypische Neuroleptika in der Behandlung der akuten Manie

Eine Alternative stellt der Einsatz atypischer Antipsychotika dar, die bei vergleichbarer Wirksamkeit ein geringeres Potential zur Auslösung von EPS haben. Bisher sind mit Olanzapin, Risperidon und Quetiapin bereits drei atypische Antipsychotika zur Therapie der bipolaren Manie zugelassen. Alle Substanzen werden inzwischen verbreitet und mit gutem Erfolg zur Behandlung der bipolaren Störung eingesetzt; insbesondere zur Akutbehandlung ist die Studienlage für Atypika gut. So konnte beispielsweise für Olanzapin in einer doppelblinden Unter- suchung eine signifikante Überlegenheit gegenüber Plazebo bei manischen Patienten nachgewiesen werden (15); für Risperidon fand sich eine äquivalente Wirksamkeit bei einem doppelblinden Vergleich mit Lithium und Haloperidol (14). Der Einsatz von Atypika bei akuter Manie stellt eine relativ sichere Behandlungsoption dar, mit der Symptome wie Unruhe, Aggressivität und Wahnideen effektiv behandelt werden können. Verbreitet werden Antipsychotika schon zu Beginn der Behandlung mit Stimmungsstabilisierern kombiniert. Diese Kombinationsbehandlung ist in der initialen Phase der Manietherapie effektiver als Lithium oder Antiepileptika alleine und ermöglicht einen schonenden Übergang auf eine kontinuierlich eingenommene Phasenprophylaxe (13). Auch bei Atypika limitieren gelegentlich unerwünschte Medikamentenwirkungen die Möglichkeit eines längerfristigen Einsatzes. So verursacht Olanzapin bei nicht wenigen Patienten eine erhebliche Gewichtszunahme; Risperidon kann dosisabhängig EPS und insbesondere Akathisie verursachen.

\section{Aripiprazol: ein partieller Dopaminagonist}

Das Chinolonderivat Aripiprazol, das in den USA bereits seit über einem Jahr zugelassen ist und dessen Zulassung in Deutschland und Europa noch in diesem Jahr erwartet 
wird, ist ebenfalls ein atypisches Antipsychotikum, dessen Wirkmechanismus sich aber entscheidend von dem aller bisherigen Neuroleptika unterscheidet (11). Klassische Neuroleptika wie Haloperidol sind im Wesentlichen Antagonisten am Dopamin-2-Rezeptor. Die meisten Atypika blockieren ebenfalls diesen Rezeptor sowie eine ganze Reihe anderer Rezeptortypen mit unterschiedlicher Affinität. Aripiprazol ist hingegen ein partieller $\mathrm{D}_{2}$-Agonist (2). Die Substanz bindet mit hoher Affinität an $\mathrm{D}_{2}$-Rezeptoren und blockiert diese gegen eine Aktivierung durch endogenes Dopamin. Im Gegensatz $z u$ reinen $D_{2}$-Antagonisten wie Haloperidol hat Aripiprazol jedoch eine intrinsische Wirksamkeit am Rezeptor und aktiviert diesen. Als partieller Agonist hat die Substanz aber keine $100 \%$ ige Aktivität und liegt in ihrer Effektivität am Rezeptor deutlich unter der von Dopamin. Auch bei hoher Besetzung fast aller striataler $\mathrm{D}_{2}$-Rezeptoren kommt es immer noch zu einer mäßigen Stimulation dieser Rezeptoren. Aripiprazol ist demnach in der Lage, eine Überstimulation dopaminerger Rezeptoren durch einen Überschuss von endogenem Dopamin zu verhindern, hält aber gleichzeitig eine moderate Rezeptoraktivität aufrecht. Aus diesen Rezeptoreigenschaften erklärt sich vermutlich der Wirkmechanismus von Aripiprazol bei der Schizophrenie. Bei dieser Erkrankung kommt es zu einer dopaminergen Dysfunktion in verschiedenen Arealen des ZNS. Im mesolimbischen System werden durch eine vermehrte Dopaminfreisetzung produktiv-psychotische Symptome verursacht (6). Diesen hyperdopaminergen Zustand blockieren alle Neuroleptika einschließlich Aripiprazol. Gleichzeitig kommt es jedoch im mesokortikalen System zu einer verminderten Aktivität der dopaminergen Transmission, die für die schizophrene Negativsymptomatik verantwortlich gemacht wird (4). Aripiprazol kann durch seinen partiellen Agonismus hier eine dopaminerge Aktivierung aufrechterhalten - im Gegensatz zu reinen $\mathrm{D}_{2}$ Antagonisten, die klinisch dementsprechend auch keine Wirksamkeit

\section{Abb. 1 Aripiprazol ist ein partieller Dopamin-Agonist}

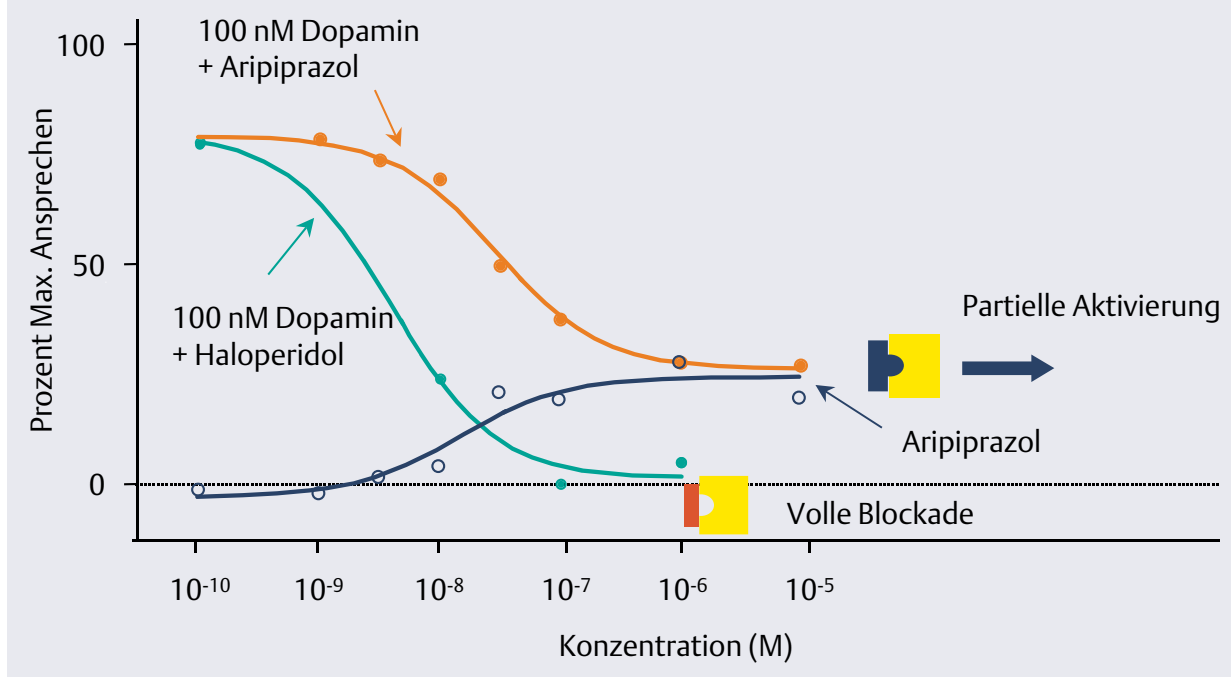

Die Abbildung zeigt Ergebnisse einer in-vitro-Untersuchung an mit humanen $D_{2}$-Rezeptoren transfizierten Zellkulturen. Auf der y-Achse ist das Ausmaß der Antwort auf dopaminerge Stimulation angegeben, die durch die Hemmung der durch Forskolin aktivierten Adenylatcyclase-Aktivität gemessen wird. Auf der x-Achse ist die Dosierung der jeweiligen Substanz aufgetragen. Dopamin in steigender Konzentration führt zu einer typischen sigmoiden Dosis-Wirkungskurve. Haloperidol alleine hat keinen Effekt auf die dopaminerge Antwort. Eine fixe Konzentration von Dopamin zusammen mit steigenden Haloperidol-Dosierungen führt zu einer bis auf Null abnehmenden dopaminergen Aktivität. Aripiprazol alleine aktiviert im Gegensatz zu Haloperidol den Dopamin-Rezeptor, jedoch in einem geringeren Ausmaß als Dopamin. In Gegenwart von Dopamin wird durch ansteigende Konzentrationen von Aripiprazol die dopaminerge Aktivität gehemmt, jedoch nicht bis auf den Nullpunkt, sondern bis zum Niveau der intrinsischen Aktivität von Aripiprazol. Die Substanz aktiviert also den $D_{2}$-Rezeptor und blockiert gleichzeitig eine übermäßige Stimulation durch Dopamin (2)

gegen die schizophrene Negativsymptomatik aufweisen (Abb. 1). Im nigrostriatalen System werden durch eine Blockade von $\mathrm{D}_{2}$-Rezeptoren durch klassische Neuroleptika die extrapyramidalmotorischen $\mathrm{Ne}$ benwirkungen verursacht. EPS kann durch den partiellen Agonismus von Aripiprazol in diesem System ebenfalls weitgehend verhindert werden. Vergleichbar verhält sich Aripiprazol am serotonergen $5-\mathrm{HT}_{1 \mathrm{~A}}$-Rezeptor, der eine wichtige Rolle in der Pathophysiologie von Depression und Angst spielt. Auch hier ist die Substanz ein partieller Agonist, jedoch mit geringerer Affinität als beim $D_{2}$ Rezeptor (7).

\section{Aripiprazol ist wirksam in der Behandlung der Schizophrenie}

Die Wirksamkeit von Aripiprazol in der Behandlung der Schizophrenie wurde in mehreren Kurz- und Langzeitstudien nachgewiesen. In zwei doppelblinden Studien über jeweils 28 Tage zeigte Aripiprazol eine signifikant bessere Wirkung als Plazebo und war gleich wirksam wie Haloperidol und Risperidon $(8,12)$. Über einen Zeitraum von einem halben Jahr verdoppelte Aripiprazol im Vergleich zu Plazebo die Dauer bis zu Auftreten eines Rezidivs und erwies sich nach einem Jahr dem Haloperidol deutlich überlegen (10).

\section{Therapie der Manie mit Aripiprazol}

Aufgrund der Stabilisierung sowohl des Dopamin- als auch des Serotoninsystems durch Aripiprazol verspricht die Substanz eine mögliche bipolare Wirkung: Wie $\mathrm{D}_{2}$-Antagonisten könnte Aripiprazol einer dopaminergen Überfunktion und damit einer manischen Exazerbation entgegenwirken, während durch die Blockade von 5- $\mathrm{HT}_{1 \mathrm{~A}}-\mathrm{Re}$ zeptoren möglicherweise eine antidepressive Wirkung zu erwarten 


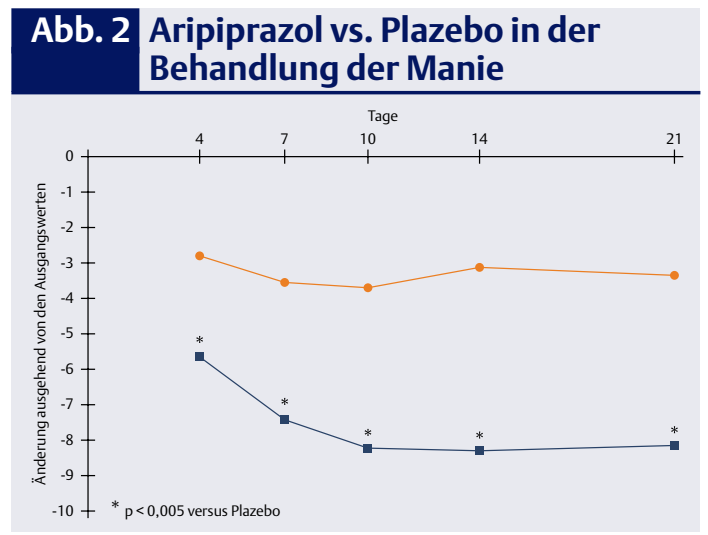

Bei 262 Patienten mit akuter Manie führte Aripiprazol über 3 Wochen zu einer signifikant größeren Abnahme der Young Mania Rating Skala als Plazebo (9)
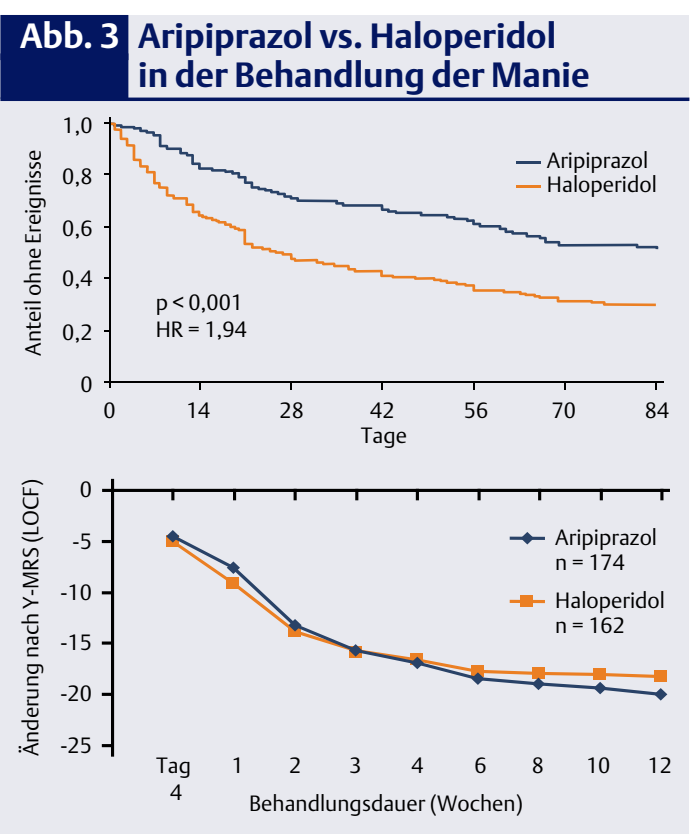

In einer 12 Wochen dauernden Studie wurden 347 manische Patienten untersucht und doppelblind mit Aripiprazol oder Haloperidol behandelt. Die Gruppen unterschieden sich nicht signifikant hinsichtlich der Verringerung der Young Mania Skala, jedoch in der Zeit bis zum Studienabbruch, der hauptsächlich durch unerwünschte Arzneimittelwirkungen verursacht wurde (1)

wäre (5). Es lag daher nahe, die Wirksamkeit von Aripiprazol neben der Schizophrenie auch bei affektiven Erkrankungen zu testen.

Bislang liegen die Ergebnisse von zwei großen Multicenter-Studien vor, die den Einsatz von Aripiprazol bei akuten Manien untersuchten. Die erste Untersuchung wurde in 38 US-amerikanischen Zentren durchgeführt (9). In diese Studie wurden
262 Patienten eingeschlossen; es handelt sich damit um die größte bislang durchgeführte plazebo-kontrollierte Maniestudie. In der 3wöchigen Untersuchung wurden die Patienten mit einer Einmalgabe von $30 \mathrm{mg}$ Aripiprazol, das bei Unverträglichkeit auf $15 \mathrm{mg}$ reduziert werden konnte, oder Plazebo behandelt. Die Behandlung musste in den ersten beiden Wochen unter stationären Bedingungen durchgeführt werden. Nach Ende der zweiten Woche konnten die Patienten bei Besserung der Symptomatik entlassen werden; bei klinischer Verschlechterung konnte zu diesem Zeitpunkt auf eine offene Behandlung mit dem Verum umgestellt werden. Visiten wurden an den Tagen 4, 7, 10, 14 und 21 durchgeführt; primäres Zielkriterium war die Veränderung des Wertes in der Young Mania Rating Scale (YMRS), der bei Studienbeginn bei durchschnittlich 29 lag. Als Komedikation konnte Lorazepam in relativ hoher Dosis verwendet werden.

In der Aripiprazol-Gruppe beendeten $42 \%$ der Patienten die dreiwöchige doppelblinde Behandlungsphase, in der Plazebogruppe nur $21 \%$. Bei etwa $10 \%$ der Patienten pro Behandlungsgruppe musste die Studie wegen unerwünschter Nebenwirkungen abgebrochen werden, mangelhafte Wirkung war hingegen der Hauptgrund für die übrigen, zwischen den Gruppen signifikant unterschiedlich häufigen Abbrüche. Die durchschnittliche Aripiprazol-Dosierung war 27,9 mg pro Tag; bei 86\% der Patienten wurde die anfängliche Dosis von 30 mg pro Tag bis zum Studienende weitergeführt.

Die Unterschiede der YMRSWerte waren von Tag 4 an signifikant (Abb. 2). Aripiprazol führte bei Studienende zu einer Verminderung des YMRS-Wertes um 8,15 Punkte; Plazebo reduzierte den Ausgangswert hingegen nur um 3,35 Punkte. Unter Aripiprazol wurden nach drei Wochen 40\% der Patienten als Responder eingestuft, unter Plazebo nur 19\%. Auch bei Verwendung der Clinical Global Impression Skala (CGI) war Aripiprazol signifikant überlegen. Gastrointestinale Nebenwirkungen, Akathisie und Sedierung wurden in der Aripiprazolgruppe häufiger als in der Kontrollgruppe als unerwünschte Wirkungen berichtet. Die meisten Nebenwirkungen traten in der ersten Behandlungswoche auf und hielten nur wenige Tage an. In zwei Skalen, die extrapyramidal-motorische Nebenwirkungen erfassten, kam es unter Aripiprazol zwar nur zu marginalen, aber signifikanten Verschlechterungen im Vergleich zu Plazebo. Unter beiden Behandlungsregimen kam es zu einer leichten Gewichtsabnahme und durchschnittlich zu einer Verminderung des Prolaktinspiegels. Eine Veränderung des QT-Intervalls oder hämatologische Komplikationen wurden unter Aripiprazol nicht beobachtet.

Gemäß den US-amerikanischen Zulassungsbestimmungen wurde in dieser Studie die Überlegenheit von Aripiprazol gegenüber Plazebo nachgewiesen. Die Wirksamkeitsparameter entsprechen in ihrer Größenordnung den Ergebnissen ähnlicher Studien mit anderen Atypika; es wurde ein schneller Wirkungseintritt innerhalb weniger Tage beobachtet. Aripiprazol wurde generell gut vertragen, so dass es zu wenigen Behandlungsabbrüchen aufgrund unerwünschter Wirkungen kam.

Ein anderes Design wählte eine zweite Studie, die hauptsächlich in Europa durchgeführt wurde (1). In diese Studie wurden 347 Patienten mit einer manischen oder gemischten Episode eingeschlossen, die über zwölf Wochen behandelt wurden. Aripiprazol wurde in einer Dosierung von $15 \mathrm{mg} /$ Tag begonnen und konnte bis auf $30 \mathrm{mg}$ erhöht werden, die durchschnittliche Dosierung bei Studienende betrug 21,6 mg/Tag. Als Vergleichssubstanz diente in dieser Untersuchung Haloperidol, das von 10 auf $15 \mathrm{mg} /$ Tag titriert werden konnte. Hier betrug die durchschnittliche Dosierung 11,6 mg/Tag. Primäres Zielkriterium war die Responder-Rate.

50,9\% der Patienten unter Aripiprazol und 29,1\% der Patienten unter Haloperidol beendeten die zwölfwöchige Behandlungsphase. Etwa 50\% der Aripiprazol-Patienten wurden nach zwölf Wochen aufgrund einer Reduktion des YMRS um min- 
destens 50\% als Responder eingestuft, in der Haloperidol-Gruppe jedoch nur knapp 30\%. Dieser Unterschied war signifikant. Beide Gruppen unterschieden sich hinsichtlich der Abnahme des YMRS-Wertes nur wenig, aber es kam zu einer wesentlich höheren Responder-Rate unter Aripiprazol aufgrund der sehr viel kleineren Zahl von Studienabbrechern (Abb. 3). Hauptgrund für die Beendigung der Therapie waren unerwünschte Wirkungen, insbesondere EPS und Akathisie, die unter Haloperidol sehr viel häufiger auftraten. In der Haloperidolgruppe stiegen die Prolaktinspiegel an, unter Aripiprazol fielen sie ab. Auch in dieser Studie wurden weder Gewichtszunahme noch EKG-Veränderungen beobachtet.

Im Gegensatz zur US-amerikanischen Zulassungsstudie entspricht diese Untersuchung eher der europäischen Behandlungsrealität, da gegen Haloperidol getestet wurde und ein längerer Behandlungszeitraum gewählt wurde. Auch wurden keine Rapid-Cycling-Patienten eingeschlossen, die durch eine erhöhte Rate von Spontanremissionen die Ergebnisse möglicherweise verzerren könnten. Die Wirksamkeit von Aripiprazol entsprach der von Haloperidol, die Substanz wurde jedoch weitaus besser vertragen, so dass die Behandlung bei mehr Patienten über einen längeren Zeitraum fortgeführt werden konnte.

Zusammenfassend ist Aripiprazol eine Substanz, die über eine ausgeprägte antimanische Wirksamkeit bei guter Verträglichkeit verfügt. Ihr Einsatz bietet sich bei solchen Patienten an, deren manische Symptomatik alleine durch Stimmungsstabilisierer wie Lithium oder Valproat nicht unter Kontrolle zu bekommen ist. Es scheint zu weniger EPS als unter klassischen Neuroleptika und zu einer geringeren Gewichtszunahme als unter Olanzapin zu kommen. Erstmals wurde ein Atypikum primär noch vor der Markteinführung für die Indikation der Schizophrenie im großen Maßstab an bipolaren Patienten mit dem Ziel der Zulassung für diese Indikation gestestet. Die Ergebnisse von Langzeitstudien zur Phasenprophylaxe und zur Behandlung bipolarer Depressionen stehen noch aus. Dennoch handelt es sich bei Aripiprazol schon jetzt um eine vielversprechende $\mathrm{Er}$ weiterung der medikamentösen Möglichkeiten bei bipolaren Erkrankungen.

In the past most manic patients were treated with classical antipsychotic substances, but these are increasingly being superseded by atypical agents. Particularly in combination with mood-stabilising drugs, atypical antipsychotics offer an effective and largely safe treatment option in acute manic exacerbations. Aripiprazole is an atypical antipsychotic with a novel mechanism of action. The substance is a partial dopamine agonist at the $D_{2}$ receptors where it prevents dopaminergic overstimulation without switching off the receptors entirely. Aripiprazole also acts as a partial agonist at $5-H T_{1 A}$ receptors, whereby the effect at $5-\mathrm{HT}_{2 \mathrm{~A}}$ receptors is antagonistic. Aripiprazole has already been licensed in the US for the treatment of schizophrenia, and the European marketing authorisation has been applied for.

Two major double-blind studies were conducted to investigate aripiprazole in the treatment of acute mania.

These showed it to be superior to placebo and equally effective as haloperidol. Treatment with aripiprazole was better tolerated than with haloperidol, and there were fewer dropouts. Aripiprazole appears to be a most promising therapy option for bipolar disorders.

Key Words: Bipolar Mania - Aripiprazole - partial agonism - schizophrenia

\section{Literatur}

1. Bourin M, Auby P, Swanink R, Marcus R, McQuade RD, Iwamoto T, Sanchez R. Aripiprazole vs haloperidol for maintained treatment effect in acute mania. (poster) APA Congress; 2003

2. Burris KD, Molski TF, Xu C, Ryan E, Tottori K, Kikuchi T, Yocca FD, Molinoff PB. Aripiprazole, a novel antipsychotic, is a high-affinity partial agonist at human dopamine $D_{2}$ receptors. J Pharmacol Exp Ther 2002: 302; 381-389

3. Colom F, Vieta E, Martinez-Aran A, Reinares M, Benabarre A, Gasto C. Clinical factors associated to treatment non-compliance in bipolar disorder. J Clin Psychiatry 2000; 61: 549-554
4. Deutch AY. The regulation of subcortical dopamine systems by the prefrontal cortex: interactions of central dopamine systems and the pathogenesis of schizophrenia. J Neural Transm Suppl 1992; 36: 61-89 5. Diehl DJ, Gershon S. The role of dopamine in mood disorders. Compr Psychiatry 1992; 33: 417-8

6. Goldstein M, Deutch AY. Dopaminergic mechanisms in the pathogenesis of schizophrenia. FASEB J 1992; 6: 2413-21

7. Jordan S, Koprivica V, Chen R, Tottori K, Kikuchi T, Altar CA. The antipsychotic aripiprazole is a potent, partial agonist at the human 5-HT1A receptor. Eur J Pharmacol 2002; 441:137-40

8. Kane JM, Carson WH, Saha AR, McQuade RD, Ingenito GG, Zimbroff DL, Ali MW. Efficacy and safety of aripiprazole and haloperidol versus placebo in patients with schizophrenia and schizoaffective disorder. J Clin Psychiatry 2002; 63: 763-71

9. Keck PE, Marcus R, Tourkodimitris S, Ali M, Liebeskind A, Saha A, Ingenito G. A placebo-controlled, double-blind study of the efficacy and safety of aripiprazole in patients with acute bipolar mania. Am J Psychiatry 2003;160(9): 1651-8

10. Kujawa M, Saha A, Igenito G, Ali M. Aripiprazole for long-term maintenance treatment of schizophrenia (Poster) XXIII CINP Congress 2002

11. McGavin JK, Goa KL. Aripiprazole. CNS Drugs 2002; 16: 779-786

12. Potkin SG, Saha AR, Kujawa MJ, Carson WH, Ali M, Stock E, Stringfellow J, Ingenito G, Marder SR. Aripiprazole, an antipsychotic with a novel mechanism of action, and Risperidone vs placebo in patients with schizophrenia and schizoaffective disorder. Arch Gen Psychiatry 2003; 60: 681-690

13. Sachs GS, Grossman F, Ghaemi SN, Okamoto A, Bowden CL. Combination of a mood stabilizer with risperidone or haloperidol for treatment of acute mania: a doubleblind, placebo-controlled comparison of efficacy and safety. Am J Psychiatry 2002; 159 : 1146-1154

14. Segal J, Berk M, Brook S. Risperidone compared with both lithium and haloperidol in mania: a double-blind randomized controlled trial. Clin Neuropharmacol 1998; 21: $176-180$

15. Tohen M, Sanger TM, McElroy SL, Tollefson GD, Chengappa KN, Daniel DG, Petty F, Centorrino F, Wang R, Grundy SL, Greaney MG, Jacobs TG, David SR, Toma V. Olanzapine versus placebo in the treatment of acute mania. Olanzapine HGEH Study Group. Am J Psychiatry 1999; 156: 702-709 16. Zarate CA, Tohen M. Antipsychotic drug treatment in first-episode mania: a 6-month longitudinal study. J Clin Psychiatry 2000; 61:33-38

\section{Korrespondenzadresse:}

Dr. Claus Normann

Abtl. Psychiatrie und Psychotherapie mit Poliklinik

Hauptstr. 5

79104 Freiburg 